\title{
Huge Broad Ligament Leiomyoma: A Case Report
}

\author{
Fatima Bakari*, Hajaratu U. Sulayman, Solomon Avidime, Nkeirukah Ameh, \\ Adebiyi G. Adesiyun \\ Department of Obstetris and Gynaecology, Ahmadu Bello University Teaching Hospital, Zaria, Nigeria \\ Email: ${ }^{*}$ fadimatubakar@gmail.com
}

Received 19 January 2015; accepted 1 February 2015; published 5 February 2015

Copyright (C) 2015 by authors and Scientific Research Publishing Inc.

This work is licensed under the Creative Commons Attribution International License (CC BY). http://creativecommons.org/licenses/by/4.0/

\section{Abstract}

The broad ligament is the commonest extra uterine site for the occurrence of leiomyoma but with a very low incidence rate. Because of its overall low incidence rate, it poses both clinical and radiological difficulty in differentiating with an ovarian tumour. We report a case of a 31-year-old unmarried nullipara (para $0+0$ ) who presented with 12-month history of progressive abdominal swelling, three-month history of pelvic pain and one-month history of prolonged heavy menstrual flow. Examination of the abdomen showed a 36-week-size mass which was multi-lobulated, firm, non tender and slightly mobile extending to the xyphisternum. On abdominal ultrasonography scan, the uterus was deviated to the contra lateral side with a huge, irregular mass measuring $24 \times$ $18 \mathrm{~cm}$ posterior to the uterus. She had laparotomy and four fibroid seedlings were removed. The largest was a broad ligament myoma which measured $24 \times 17 \times 12 \mathrm{~cm}$ and weighed $3883 \mathrm{~g}$.

\section{Keywords}

Broad Ligament, Leiomyoma, Myomectomy

\section{Introduction}

Leiomyoma is generally the most common benign tumour of the uterus and the female genital tract [1]. This benign tumour commonly arises from the smooth muscle of the uterus but occasionally, they arise in smooth muscles at extra uterine site. Broad ligament is the most common extra uterine site for the occurrence of leiomyoma [2]; the incidence is however $<1 \%$ [3]. Other extra uterine sites are the round ligament, ovarian ligament and the ovaries. They are sometimes found at distant sites such as the lungs [4]. Leiomyoma in the broad ligament has been documented to reach an enormous size which can mimic ovarian malignancy [5]. It often coexists with fibroid in the uterus [1].

${ }^{*}$ Corresponding author. 
These benign tumours in the broad ligament are usually asymptomatic but if neglected for a long time, it can reach an enormous size resulting in chronic pelvic pain, compression of contiguous structures like the bladder and the bowel and resulting in bladder and bowel dysfunction. It can lead to menstrual abnormalities especially with a coexisting intrauterine myoma.

This case was reported because of the rare incidence of broad ligament leiomyoma and the difficulty one may face in differentiating it from an ovarian tumour. Ovarian malignancy should be considered prior to a planned surgery.

\section{Case Report}

A 31-year-old single nulliparous housewife presented to the Gynaecology Clinic with complaints of mass in her abdomen of one-year duration, abdominal pain of three months duration and prolonged menstrual period of one month duration. The mass was first noticed as a small lump in the lower part of her abdomen and gradually progressed to reach an enormous size above the umbilicus. She developed dull achingabdominal pain associated with heaviness in the lower part of her abdomen. There were no disturbances in her bladder and bowel function. There was history of prolonged heavy menstrual flow that started one month prior to presentation. Bleeding became scanty following ingestion of medication obtained from a pharmacy. There was no history of weight loss or jaundice.

She had history of fibroid in her mother and younger sister but had no family history of ovarian, breast or colonic tumour.

General physical examination revealed an average sized woman with mild pallor. She had no pedal oedema and there was no significant lymphadenopathy. Her pulse rate was 76 beat per minute, regular and full volume. Her blood pressure was 110/70 mmHg. Her chest was clinically clear. Her abdomen was distended with a huge mass to a pregnancy size of 36 weeks. There were no distended anterior abdominal wall veins. The mass was arising from the pelvis and encroaching laterally to the paracolic gutters. A smaller mass that felt separate was palpated in the left iliac fossa. The masses were non tender and firm to hard in consistency with bumpy surfaces. It has limited mobility from side to side. The liver and spleen were not enlarged. Ascitis was not demonstrated. Pelvic examination revealed normal vulva and vagina. The cervix was drawn up to the left side. It was closed and grossly looking healthy. The smaller mass in the left iliac fossa moved with movement of the cervix.

She had an abdominal ultrasound which showed a huge solid hypo echoic irregular mass measuring $24 \times 22$ $\mathrm{cm}$ occupying the postero-lateral aspect of the uterus. The uterus was seen anterior and inferior to the mass. Smaller myoma masses were noted within the myometrium. There was mild pelvicaliceal dilatation of the right kidney. There was no lymphadenopathy noted. Her packed cell volume was 30\%. Her full blood count and differential, and serum biochemistry was within normal limits. CA 125 was slightly above the upper limit. CT scan and IVU were not done because of patient's financial constraint. A diagnosis of leiomyoma to rule out ovarian tumour was made. She was worked up for laparotomy.

Intraoperatively, following a midline longitudinal incision with extension over the umbilicus, a huge right broad ligament leiomyoma with adherent extension to the posterior wall of the uterus and measuring approximately $25 \times 26 \mathrm{~cm}$ in its widest diameter was seen. The uterus was noted to be bulky, pushed up and displaced to the left side. Three smaller intramural fibroid were noted on the anterior and posterior aspect of the uterus. The right fallopian tube and right ovary was stretched over the mass but looked grossly normal. The left fallopian tube was crowded and buried in adhesion with the left ovary. See Figure 1.

A tourniquet was tied to the cervico-uterine junction of the uterus before removal of the fibroid seedlings to reduce blood loss at surgery. The endometrium was breached posteriorly while removing the posterior intramural seedling. The exposed endometrial cavity was closed in two layers. The myometrial and serosal layers of the uterus was repaired using Monocryl 1 suture, ensuring haemostasis. See Figure 2.

The estimated intraoperative blood loss was $400 \mathrm{ml}$. A Size 8 Foley's urethral catheter was inserted into the uterine cavity and the balloon filled with $3 \mathrm{ml}$ of distilled water following the surgery to guard against the formation of intrauterine synechia.

Her post-operative packed cell volume was $26 \%$. She had 2 units of blood transfused postoperatively. She was discharged on the $10^{\text {th }}$ postoperative day following removal of the intrauterine balloon catheter.

Histopathology reported four grey white masses with the largest measuring $24 \times 17 \times 12 \mathrm{~cm}$ and the smallest measuring $3 \times 2 \times 1 \mathrm{~cm}$. The largest weighed $3883 \mathrm{~g}$. Cut sections showed grey white solid areas with whorled appearance. 


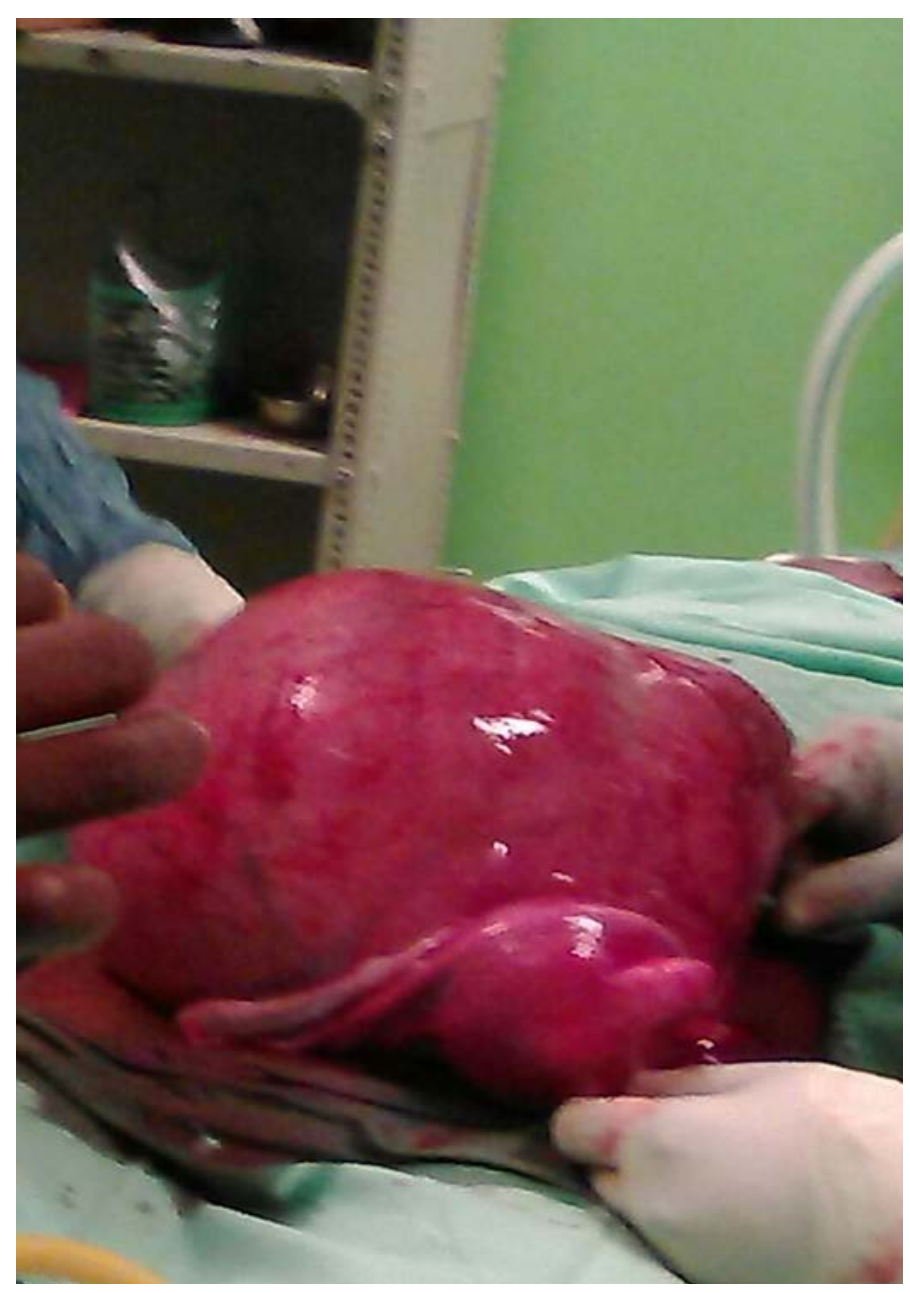

Figure 1. Broad ligament fibroid showing the uterus deviated to the left and the right fallopian tube and ovary stretched.

Microscopic examination shows an encapsulated lesion composed of interlacing smooth muscle bundle arranged in haphazard fashion. It is composed of spindle cells having bland nuclei and moderate cytoplasm. The stroma is scanty and fibrous. The pathological diagnosis was leiomyoma.

\section{Discussion}

Broad ligament leiomyoma is a benign smooth muscle tumour which may originate primarily from the broad ligament hormone sensitive smooth muscle or secondarily from the uterine smooth muscle [5].

The location of fibroid often determines the various clinical symptoms [6]. Extra uterine leiomyoma which commonly occurs in the broad ligament are usually asymptomatic. Broad ligament fibroid have the potential to grow to a very large size [7]. If allowed to reach an enormous size, it can present with pressure symptoms of pelvic pain and bladder and bowel dysfunction. Intra uterine fibroid on the other hand in addition to pressure symptoms often presents with menstrual abnormalities and dysmenorrhoea. The case presented here had both pressure symptoms of chronic lower abdominal pain and heaviness in the lower abdomen likely to have been caused by the huge broad ligament fibroid and menstrual abnormalities likely to be due to the intrauterine fibroid she had.

Enlargement of the leiomyoma can cause upward displacement of the uterus and it can become impacted in the pelvis leading to ureteric obstruction, urinary retention and or constipation. This patient had huge right broad ligament myoma that pushed her uterus upward and to the left side resulting in right hydronephrosis and hydroureter. She did not however present with obstructive urinary symptoms and constipation. 


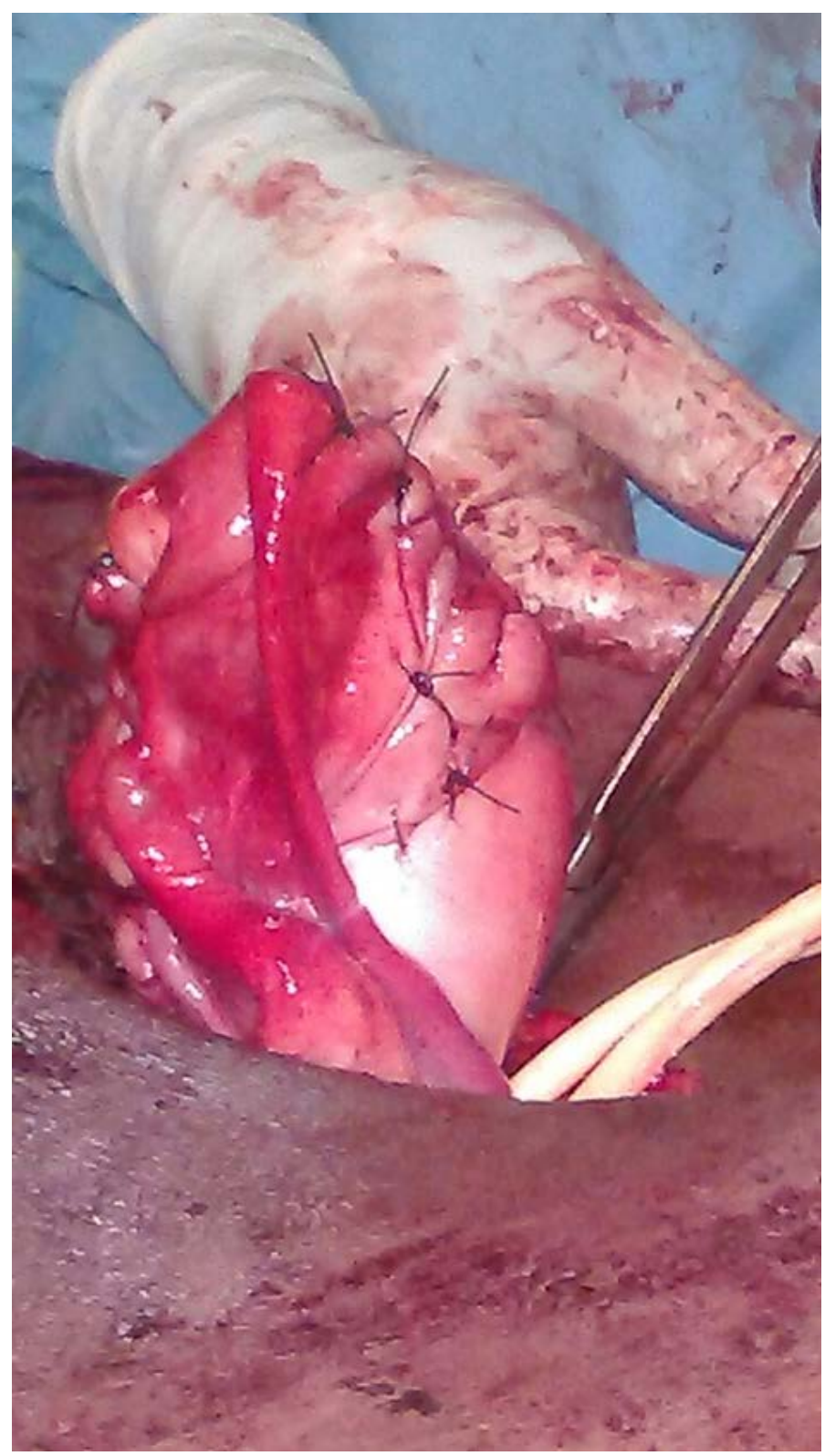

Figure 2. Post myomectomy uterus showing the right fallopian tube and ovary.

Uterine leiomyoma usually does not pose clinical and sonological diagnostic challenges. Radiological investigations are often requested for in other to assess the extent of the mass and its relationship with other pelvic and abdominal structures. However, extra uterine fibroid distorting the pelvic anatomy may raise suspicion of an ovarian tumour [8]. This case reports one such circumstance where the patient presented with a mass that reached and enormous size within twelve months and with clinical and radiological features that made it necessary to rule out a possibility of ovarian malignancy. Her serum CA 125 which is a non-specific tumour marker was just marginally raised. Computerized tomography (CT) scan and intravenous urography (IVU) were outlined for her to do to aid in making a definitive diagnosis. She could not do these investigations because of her financial constraint.

With the suspicion of an ovarian tumour, she had an exploratory laparotomy. The intraoperative diagnosis was huge broad ligament myoma and multiple intrauterine myoma. The patient had an abdominal myomectomy to conserve her fertility. 


\section{Conclusion}

Huge broad ligament leiomyoma distorting the pelvic anatomy and presenting with atypical ultrasound features may pose difficulties in differentiating it with an ovarian tumour. It is also not uncommon for it to coexist with uterine myoma. Ovarian malignancy must be considered prior to a planned surgery.

\section{References}

[1] Neha, G. and Manisha, L. (2014) A Rare Case of Giant Broad Ligament Fibroid with Cervical Fibroid Mimicking Ovarian Tumour. International Journal of Recent Trends in Science and Technology, 10, 208-209.

[2] Bhatta, N. (2001) Tumours of the Corpus Uteri. Jeffcoats Principles of Gynaecology. 6th Edition, Arnold Printers, London, 470.

[3] Parker, W.H. (2005) Uterine Myoma: An Overview of Development, Clinical Features and Management. Obstetrics Gynecology, 105, 216-217. http://dx.doi.org/10.1097/01.AOG.0000150453.10906.b6

[4] Rajesh, S. and Adam, M. (2013) Benign Metastasizing Leiomyoma: “A Sheep in Wolf’s Clothing”. Community Oncology, 10, 122-125.

[5] Dayananda, K.R., Vaibhav, P., Sujid, J. and Sujatha, N.D. (2013) Broad Ligament Fibroid Mimicking as Ovarian Tumour on Ultrasonography and CT Scan. Journal of Clinical Imaging Science, 3, 8. http://dx.doi.org/10.4103/2156-7514.107912

[6] Stewart, E.A. (2001) Uterine Fibroid. Lancet, 357, 293-298. http://dx.doi.org/10.1016/S0140-6736(00)03622-9

[7] Jagtap, S.V., Gupta, A. and Kshirsaga, N.S. (2014) Broad Ligament Myoma Mimicking as Ovarian Tumour. Scholars Journal of Applied Medical Sciences, 2, 258-260.

[8] Shabeen, N., Yasir, M. and Janta, M. (2014) Diagnostic Dilemma in Broad Ligament Leiomyoma with Cystic Degeneration. Pakistan Journal of Medical Sciences, 30, 452-454. 
Scientific Research Publishing (SCIRP) is one of the largest Open Access journal publishers. It is currently publishing more than 200 open access, online, peer-reviewed journals covering a wide range of academic disciplines. SCIRP serves the worldwide academic communities and contributes to the progress and application of science with its publication.

Other selected journals from SCIRP are listed as below. Submit your manuscript to us via either submit@scirp.org or Online Submission Portal.
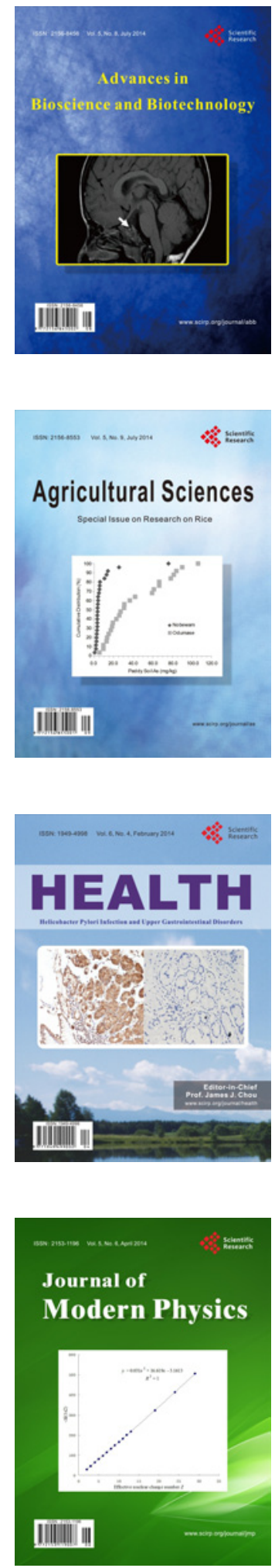
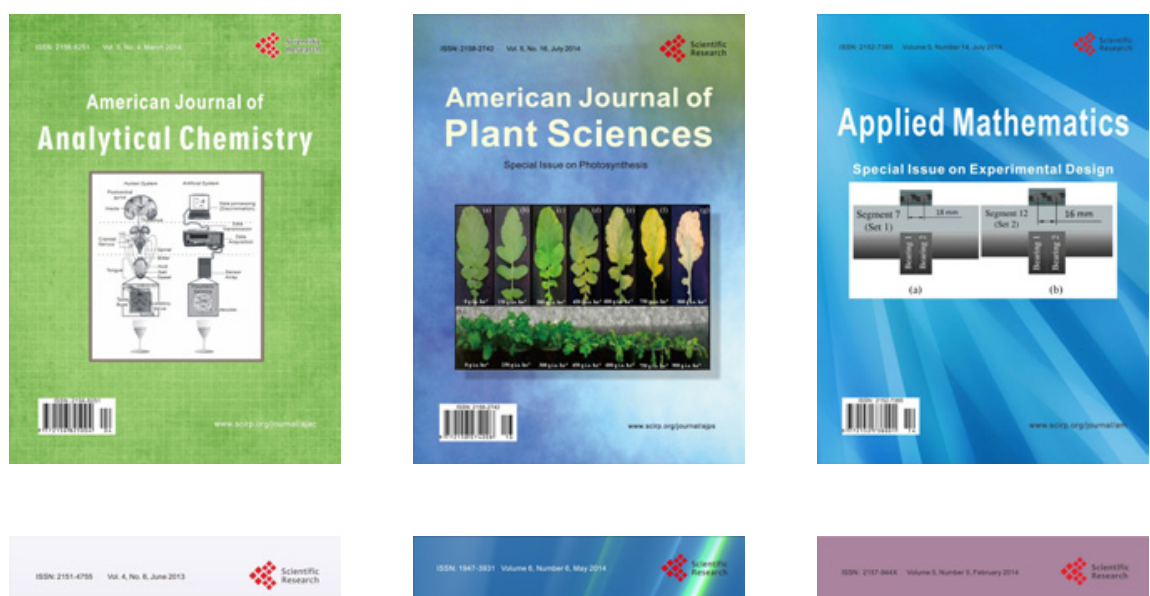

Creative Education
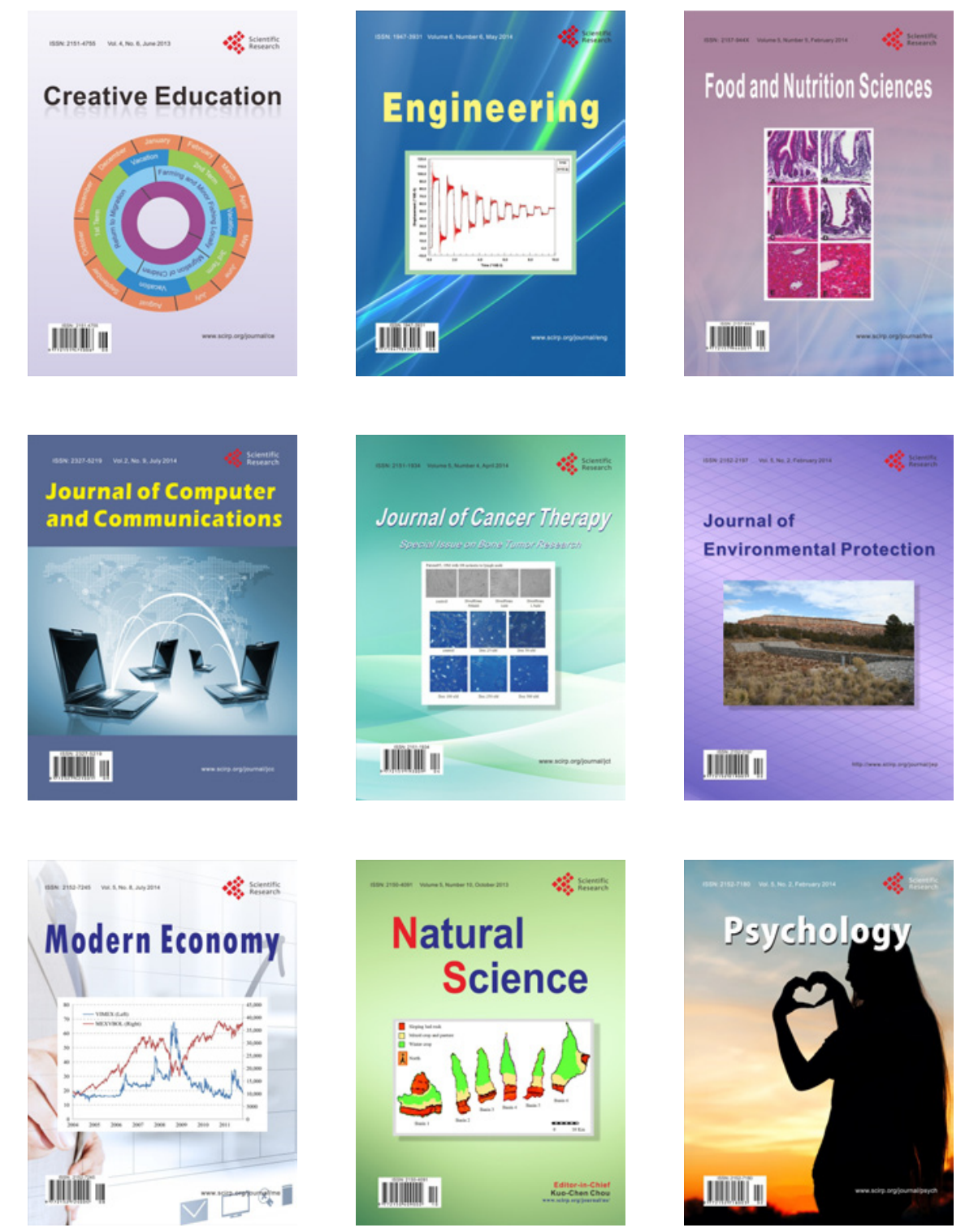